\title{
Author Correction: Quantum wave-particle superposition in a delayed-choice experiment
}

Kai Wang (D), Qian Xu, Shining Zhu and Xiao-song Ma (D)

Correction to: Nature Photonics https://doi.org/10.1038/s41566-019-0509-0, published online 2 September 2019.

In the version of this Article originally published, in the Acknowledgments, grant '11690032' from the National Natural Science Foundation of China was mistakenly not mentioned; this information has now been added to the sentence beginning "This research is supported by the.... The online versions of the Article have been amended.

Published online: 28 April 2020

https://doi.org/10.1038/s41566-020-0643-8

(c) The Author(s), under exclusive licence to Springer Nature Limited 2020 